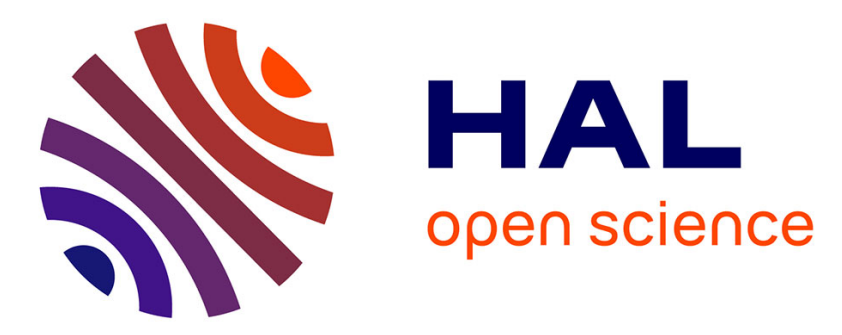

\title{
Learning overcomplete dictionaries based on parallel atom-updating
}

Mostafa Sadeghi, Massoud Babaie-Zadeh, Christian Jutten

\section{To cite this version:}

Mostafa Sadeghi, Massoud Babaie-Zadeh, Christian Jutten. Learning overcomplete dictionaries based on parallel atom-updating. MLSP 2013 - IEEE 23rd International Workshop on Machine Learning for Signal Processing, Sep 2013, Southampton, United Kingdom. 5 p. hal-00867170

\section{HAL Id: hal-00867170 https://hal.science/hal-00867170}

Submitted on 27 Sep 2013

HAL is a multi-disciplinary open access archive for the deposit and dissemination of scientific research documents, whether they are published or not. The documents may come from teaching and research institutions in France or abroad, or from public or private research centers.
L'archive ouverte pluridisciplinaire HAL, est destinée au dépôt et à la diffusion de documents scientifiques de niveau recherche, publiés ou non, émanant des établissements d'enseignement et de recherche français ou étrangers, des laboratoires publics ou privés. 


\title{
LEARNING OVERCOMPLETE DICTIONARIES BASED ON PARALLEL ATOM-UPDATING
}

\author{
Mostafa Sadeghi ${ }^{1}$, Massoud Babaie-Zadeh ${ }^{1}$, Christian Jutten ${ }^{2 *}$ \\ ${ }^{1}$ Electrical engineering department, Sharif University of Technology, Tehran, Iran. \\ m. saadeghii@gmail.com, mbzadeh@yahoo.com \\ ${ }^{2}$ GIPSA-Lab, University of Grenoble, and the Institut Universitaire de France, France. \\ christian. jutten@gipsa-lab.grenoble-inp.fr
}

\begin{abstract}
In this paper we propose a fast and efficient algorithm for learning overcomplete dictionaries. The proposed algorithm is indeed an alternative to the well-known K-Singular Value Decomposition (K-SVD) algorithm. The main drawback of K-SVD is its high computational burden especially in highdimensional problems. This is due to the fact that in the dictionary update stage of this algorithm an SVD is performed to update each column of the dictionary. Our proposed algorithm avoids performing SVD and instead uses an especial form of alternating minimization. In this way, as our simulations on both synthetic and real data show, our algorithm outperforms K-SVD in both computational burden and the quality of the results.
\end{abstract}

Index Terms - Sparse approximation, compressive sensing, dictionary learning, alternative minimization

\section{INTRODUCTION}

\subsection{Sparse Approximation}

Sparse approximation of signals has received a lot of attention during the last decade [1]. This is due to its capability in various applications such as Compressive Sensing (CS) [2], image processing tasks (e.g. denoising, compression, inpainting, zooming) [3], and linear regression and variable selection [4]. The sparse approximation problem consists in approximating a given signal as a linear combination of as few as possible basis functions. Each basis function is called an atom and the collection of them is called a dictionary [5]. This dictionary is overcomplete, i.e. the number of atoms is (much) more than the dimension of each atom. Specifically, let $\mathbf{y} \in \mathbb{R}^{n}$ be the signal whose sparsest approximation is going to be found in $\mathbf{D} \in \mathbb{R}^{n \times K}$, with $K>n$. This amounts to solve the following problem:

$$
P_{0}: \quad \min _{\mathbf{x}}\|\mathbf{x}\|_{0} \quad \text { subject to }\|\mathbf{y}-\mathbf{D} \mathbf{x}\|_{2} \leq \epsilon
$$

* This work has been partially funded by the Iran National Science Foundation (INSF). where $\|\cdot\|_{0}$ stands for the so-called $\ell_{0}$ (pseudo) norm, which counts the number of nonzero elements, and $\epsilon$ is a small positive constant. The above problem needs a combinatorial search and is generally NP-hard [6]. So, alternative methods are used to solve it $[1,7]$. One of the most successful ideas is to replace the non-convex sparsity measure $\|\cdot\|_{0}$ with its best convex approximation $\|\cdot\|_{1}$ [8] which leads to the following convex problem:

$$
P_{1}: \quad \min _{\mathbf{x}}\|\mathbf{x}\|_{1} \quad \text { subject to }\|\mathbf{y}-\mathbf{D} \mathbf{x}\|_{2} \leq \epsilon .
$$

Many algorithms have been introduced to solve the problem of finding the sparsest approximation of a signal in a given overcomplete dictionary (for a good review see [9]). These algorithms can be classified into two general categories, greedy methods such as Orthogonal Matching Pursuit (OMP) [10] and Compressive Sampling Matching Pursuit (CoSaMP) [11], and relaxation methods, which replace the combinatorial $P_{0}$ problem with a tractable one, e.g. $P_{1}$ problem. Iterative Shrinkage-Thresholding (IST) [12], Iteratively Re-weighted Least Squares (IRLS) [13], and Smoothed $\ell_{0}$ (SL0) [7] are some examples of the second category. Greedy algorithms successively choose the appropriate atoms of the dictionary that result in the greatest improvement in the quality of the approximation. Theses algorithms benefit from high speed, but their accuracy is usually less than that of the second category.

\subsection{Learning overcomplete dictionaries}

For a given class of signals, e.g. class of natural facial images, the dictionary should have the capability of sparsely representing the signals. In some applications there is a predefined and universal dictionary that is known to be wellmatched to the contents of the given class of signals, for example, the overcomplete DCT dictionary for the class of natural images. These non-adaptive dictionaries are appealing because of their simplicity and in some cases their fast computations. However, learning the atoms from some training signals would result in dictionaries with the capability of bet- 
ter matching the contents of the signals. In this way, the adaptive dictionaries would outperform the non-adaptive ones in many applications such as image denoising [14], classification tasks [15], and so on.

Most of the dictionary learning algorithms are indeed a generalization of K-means clustering algorithm [16]. While in K-means, each training signal is forced to use only one atom (called centroid), in the sparse approximation case, each signal is allowed to use more than one atom provided that it uses as fewest as possible atoms. The approach of K-means to optimize a set of atoms (called codebook) is to iteratively perform two stages. In the first stage, known as the clustering stage, training signals are assigned to their nearest (usually in the sense of $\ell_{2}$ norm) atoms. The second stage is the update of the atoms in which each atom is updated as the (weighted) average of the signals in its cluster. This procedure is repeated several times. As a generalization of this approach, dictionary learning algorithms iteratively performs the two stages of sparse approximation and dictionary update. In the first stage, which is actually the clustering of signals into a union of subspaces, the sparse approximation of each signal is computed using the current dictionary. The second stage is the update of the dictionary.

In this paper we are going to propose an efficient dictionary learning algorithm, which is indeed a good alternative to $\mathrm{K}-\mathrm{SVD}$. As will be seen in the experimental results, our proposed algorithm outperforms K-SVD in both execution time and the quality of the results.

The paper is organized as follows. In the Section 2 we briefly review the general dictionary learning problem together with K-SVD algorithm. Then in Section 3 we detail our proposed algorithm. Section 4 presents some experimental results. Finally, Section 5 concludes the paper.

\section{DICTIONARY LEARNING PROBLEM}

Consider a set of $L$ training signals in $\mathbb{R}^{n}$ as $\left\{\mathbf{y}_{i}\right\}_{i=1}^{L}$. Putting these signals as the columns of the matrix $\mathbf{Y}$, the general dictionary learning problem is then to find a sparsifying dictionary, $\mathbf{D}$, by solving the following problem:

$$
\min _{\mathbf{D} \in \mathcal{D}, \mathbf{X} \in \mathcal{X}}\|\mathbf{Y}-\mathbf{D X}\|_{F}^{2}
$$

where $\mathcal{D}$ and $\mathcal{X}$ are the admissible sets of the dictionary and the coefficient matrix, respectively. $\mathcal{D}$ is usually defined as the set of all dictionaries with unit column-norm. Since we require that each signal has a sparse approximation, $\mathcal{X}$ is the set of all matrices $\mathbf{X}$ with sparse columns. As stated previously, the general approach to solve (3) is to use alternating minimization over $\mathbf{X}$ and $\mathbf{D}$, i.e. by fixing $\mathbf{D}$ the objective function is minimized over $\mathbf{X}$, and vice versa.

With a fixed $\mathbf{D}$, the minimization of (3) over $\mathbf{X}$ is equivalent to sparsely approximating the training signals over $\mathbf{D}$. Among the various sparse coding algorithms, OMP (or its variants) is very appealing. This is due to two reasons. The first reason is the speed of OMP. The second one is its capability to be efficiently implemented in batch mode. In some applications, such as dictionary learning, in which we need to find sparse approximations of a batch of signals in the same dictionary, we can factor some common operations of OMP. This fact along with the use of Cholesky factorization results in the significant acceleration of OMP which leads to BatchOMP algorithm [17].

\subsection{K-SVD}

In the dictionary update stage of K-SVD, only one atom is updated at a time. Moreover, while updating each atom, the nonzero entries in the associated row vector of $\mathbf{X}$ is also updated. In other words, only those signals participate in updating one atom that have used it. This is in accordance with the approach of $\mathrm{K}$-means in which each atom is updated using its own cluster signals. This also prevents each row vector to be filled. In what follows, we define $\mathbf{x}(\omega)$ as a vector containing those entries of $\mathbf{x}$ that are indexed by $\omega$, and $\mathbf{E}(:, \omega)$ as a matrix containing those columns of $\mathbf{E}$ that are indexed by $\omega$. Assume that we want to update $\mathbf{d}_{i}$ along with the nonzero entries of $\mathbf{x}_{T}^{i}$, the $i$ th row of $\mathbf{X}$. We define $\omega_{i}=\left\{j: \mathbf{x}_{T}^{i}(j) \neq 0\right\}$ as the support of $\mathbf{x}_{T}^{i}$. Then the problem of updating $\mathbf{d}_{i}$ along with $\mathbf{x}_{T}^{i}\left(\omega_{i}\right)$ amounts to solve the following minimization problem:

$$
\min _{\mathbf{d}, \mathbf{x}_{r}}\left\|\mathbf{E}_{i}^{r}-\mathbf{d} \mathbf{x}_{r}\right\|_{F}^{2} \quad \text { subject to } \quad\left\|\mathbf{d}_{i}\right\|_{2}=1
$$

where $\mathbf{E}_{i}^{r}=\mathbf{E}_{i}\left(:, \omega_{i}\right)$, in which $\mathbf{E}_{i}=\mathbf{Y}-\sum_{j \neq i} \mathbf{d}_{j} \mathbf{x}_{T}^{j}$ denotes the approximation error matrix when $\mathbf{d}_{i}$ is removed, and $\mathbf{x}_{r}$ is a row vector of length $\left|\omega_{i}\right|$. The above problem actually finds the closest rank-1 approximation to $\mathbf{E}_{i}^{r}$ and can be easily solved via SVD.

\section{PARALLEL ATOM-UPDATING DICTIONARY LEARNING}

In this section we introduce our proposed algorithm. This algorithm like K-SVD is based on atom-by-atom updating. The main drawback of K-SVD is its computational burden especially in high dimensions. This is due to performing SVD for atom updating. An alternative way of solving (4) is to use the idea of alternating minimization [17]. In other words, (4) is alternatively minimized over $\mathbf{d}$ and $\mathbf{x}_{r}$. A few (e.g. 3) alternations give a fast approximation to SVD. The resulting algorithm is known as the Approximate K-SVD (AK-SVD) [17]. Although performing more alternations gives a better approximation, the average performance will not exceed the performance of the exact solution, i.e. via SVD.

We describe a different way of performing alternating minimization to update the atoms and their associated row 
vectors in the coefficient matrix. To this aim, consider the overall error matrix,

$$
\mathbf{E}=\mathbf{Y}-\left(\mathbf{A}_{1}+\mathbf{A}_{2}+\ldots+\mathbf{A}_{K}\right), \forall i: \mathbf{A}_{i}=\mathbf{d}_{i} \mathbf{x}_{T}^{i}
$$

In K-SVD (or AK-SVD), in order to update (the nonzero columns of) for example $\mathbf{A}_{i}$, the updated versions of $\mathbf{A}_{1}, \ldots, \mathbf{A}_{i}$ are used to compute $\mathbf{E}_{i}$; while $\mathbf{A}_{i+1}, \ldots, \mathbf{A}_{K}$ have not been yet updated. Keeping this point in mind, we propose to update the atoms in parallel. In other words, instead of fully updating each $\mathbf{A}_{i}$ by performing $T$ alternations between $\mathbf{d}_{i}$ and $\mathbf{x}_{r}$, we perform $T$ alternations in such a way that in each alternation all of $\mathbf{A}_{i}$ 's are partially updated (using only one alternation). In this way, in the subsequent alternations all $\mathbf{A}_{i}$ 's have been partially updated. As our experimental results in Section 4 suggest, parallel updating of the atoms may result in further accelerating the convergence rate and even the quality of the final result.

To update each $\mathbf{A}_{i}$, we need to compute the error matrix $\mathbf{E}_{i}$. It can be easily seen that this matrix can be updated as follows. The overall error matrix is firstly computed as $\mathbf{E}=$ $\mathbf{Y}-\mathbf{D X}$ using the current dictionary and coefficient matrix. Then $\mathbf{E}_{i}=\mathbf{E}+\mathbf{A}_{i}$ and after updating $\mathbf{A}_{i}$ to $\mathbf{A}_{i}^{(n e w)}$, the error matrix $\mathbf{E}$ is updated as $\mathbf{E}=\mathbf{E}_{i}-\mathbf{A}_{i}^{(n e w)}$. In this way, there is no need to completely re-compute the error matrix for each atom, but instead it is updated in a ranked-1 manner.

Algorithm 1 gives a description of the proposed algorithm, which we have called it Parallel Atom-Updating Dictionary Learning (PAU-DL) algorithm. By $\operatorname{OMP}(\mathbf{Y}, \mathbf{D}, \tau)$ we mean the sparse approximation of $\mathbf{Y}$ in $\mathbf{D}$ and with threshold $\tau$. Depending on the application at hand, $\tau$ may be the threshold on the approximation error $(\epsilon$ in (1)) or the maximum allowed number of atoms in the sparse approximation of each training signal.

\section{SIMULATIONS}

We evaluate the efficiency of our proposed algorithm and K$\mathrm{SVD}^{1}$ with three sets of experiments. In the first set we aim to evaluate the capability of the two algorithms in recovery of a known dictionary. The second experiment is on an autoregressive (AR) signal, where there is no underlying dictionary, and we just evaluate the capability of the algorithms in learning a good (i.e. sparsifying) dictionary form the training signals. To further evaluate the advantage of PAU-DL over K-SVD, we consider as the third experiment the problem of image denoising over a learned overcomplete dictionary as in [14].

Our simulations were performed in MATLAB R2010b environment on a system with $3.8 \mathrm{GHz} \mathrm{CPU}$ and $8 \mathrm{~GB}$ RAM, under Microsoft Windows 7 operating system. As a rough

\footnotetext{
${ }^{1}$ For K-SVD and OMP we have used K-SVD-Box v10 and OMP-Box v10 available at http://www.cs.technion.ac.il/ ronrubin/ software.html
}

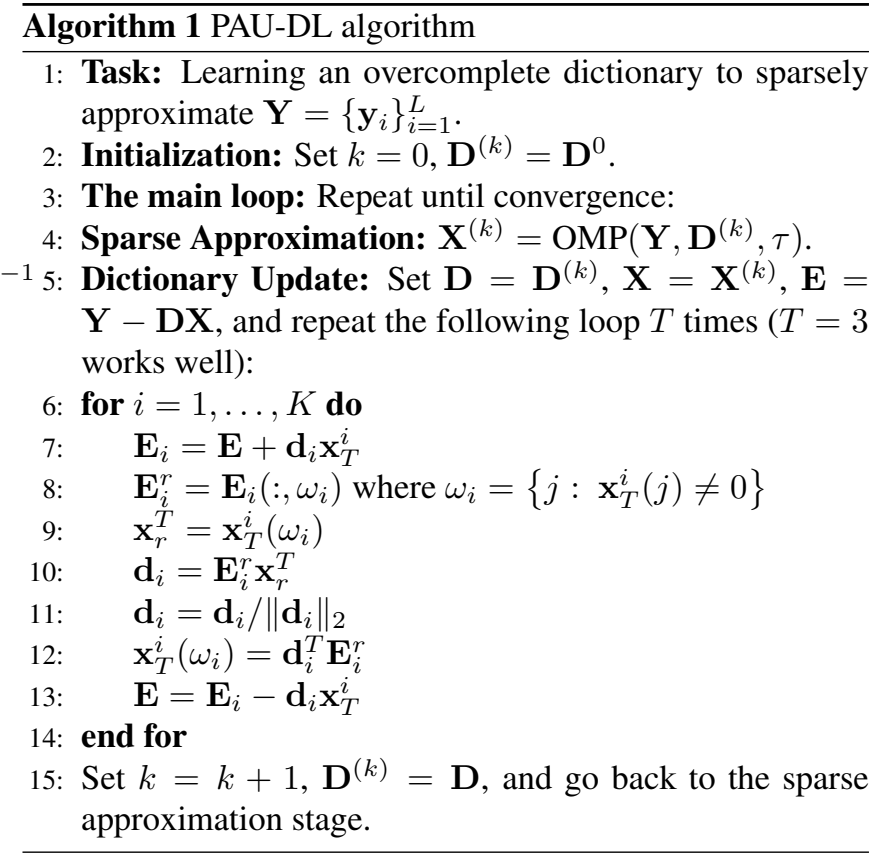

measure of complexity, we will mention the run times of the algorithms.

\subsection{Reconstruction of a known dictionary}

We generated the underlying dictionary by normalizing a random matrix of size $20 \times 50$, with zero mean and unit variance independent and identically distributed (i.i.d.) Gaussian entries. A collection of 2000 training signals $\left\{\mathbf{y}_{i}\right\}_{i=1}^{2000}$ were produced, each as a linear combination of $s$ different columns of the dictionary, with zero mean and unit variance i.i.d. Gaussian coefficients in random and independent positions. We varied $s$ from 3 to 6 . We then added white Gaussian noise with Signal to Noise Ratio (SNR) levels of 10, 20, 30, and $100 \mathrm{~dB}$. We applied all algorithms onto this noisy training signals, and compared the resulting recovered dictionaries to the generating dictionary as follows. Assume that $\mathbf{d}_{i}$ is a generating atom and $\overline{\mathbf{d}}_{i}$ is the atom in the recovered dictionary that best matches $\mathbf{d}_{i}$ among the others. We say that the recovery is successful if $\left|\mathbf{d}_{i}^{T} \overline{\mathbf{d}}_{i}\right|$ is above 0.99 [16]. The percentage of the correct recovery was used as the measure of successfully reconstructing the generative dictionary. We performed 100 alternations between sparse approximation and dictionary update stages for both algorithms. The initial dictionary was made by randomly choosing different columns of the training signals followed by a normalization.

The average percentage of successfully recovery of the underlying atoms (APSRA) is shown in Fig. 1. To see the convergence behaviour of the two algorithms, the improvement of APSRA along the alternation number for SNR = $20 \mathrm{~dB}$ and $s=4,5$ is shown in Fig. 2. The average execution times of K-SVD and PAU-DL were 27.68 and 5.41 seconds, 
respectively. With these results in mind, we deduce the following observations:

- PAU-DL has a better APSRA compared to K-SVD in average. This is especially evident at $s=5$ and $s=6$.

- The convergence rate of PAU-DL is faster than K-SVD.

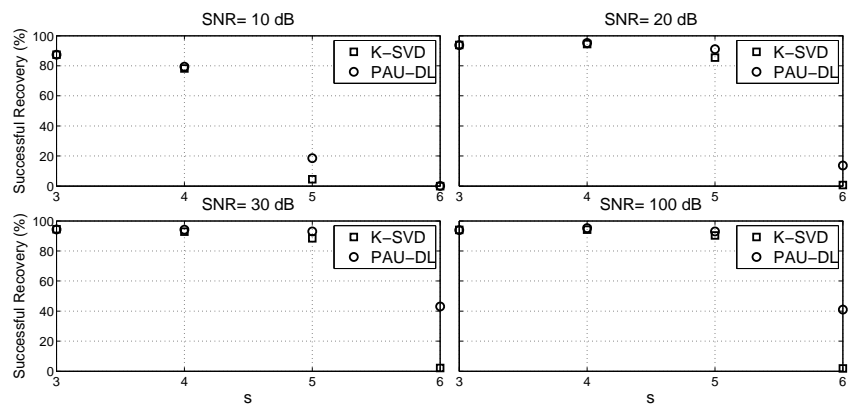

Fig. 1. Successful recovery ratios. Each figure corresponds to a certain amount of noise.
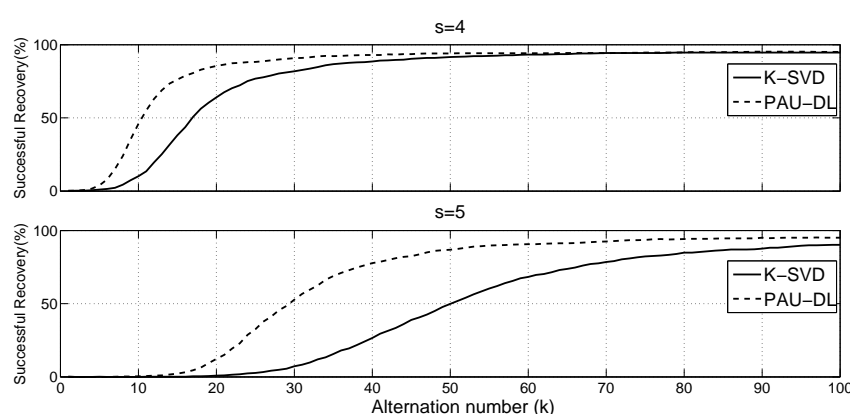

Fig. 2. Convergence behaviour of PAU-DL and K-SVD in recovery of a known dictionary $(\mathrm{SNR}=20 \mathrm{~dB})$.

\section{2. $\operatorname{AR}(1)$ signal}

In this experiment, we consider an $\mathrm{AR}(1)$ signal (according to [18]) that is generated as $v(k)=0.95 v(k-1)+e(k)$, where $e(k)$ is Gaussian noise with zero mean and unit variance. A collection of $L=2000$ training signals were made by chopping this signal into vectors of length $n=20$. Number of atoms was set to $m=40$, and $s=5$ atoms were used to approximate each training vector. For both algorithms 100 alternations were done. As in [18], we computed SNR as $\mathrm{SNR}=10 \log \|\mathbf{Y}\|_{F}^{2} /\|\mathbf{Y}-\mathbf{D X}\|_{F}^{2}$.

SNR versus alternation number is plotted in Fig. 3. From this figure we see that PAU-DL has reached a higher SNR value compared to K-SVD. The average execution times of KSVD and PAU-DL were 15.88 and 2.79 seconds, respectively.

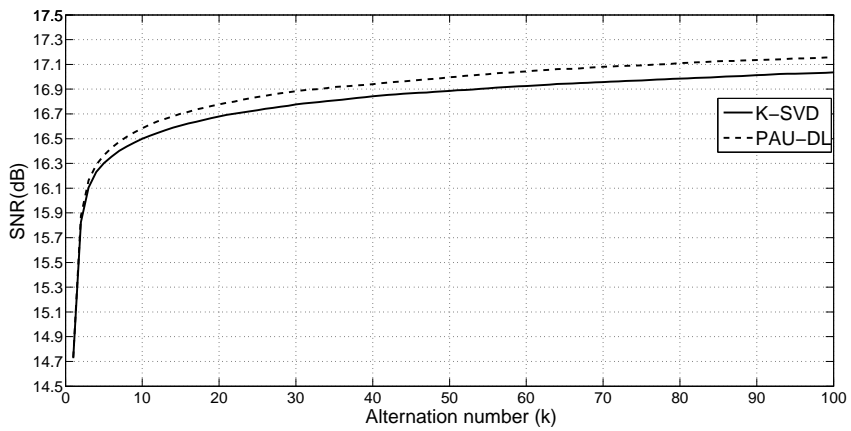

Fig. 3. $S N R$ in $d B$ is plotted versus the alternation number during the learning process.

\subsection{Image denoising over learned dictionaries}

We evaluate here the efficiency of PAU-DL relative to K-SVD in an image denoising experiment. We aim to find an estimate of a clean image contaminated with an additive white Gaussian noise with zero mean. The approach of sparse approximation is to firstly divide the noisy image into some small blocks (typically of size $8 \times 8$ ), then learn an overcomplete dictionary using these blocks as the training signals, and finally denoise each block over the learned dictionary [14]. As in [14], we used Peak Signal to Noise Ratio (PSNR), as the criterion for comparison of the denoising quality. We considered four test images, all of size $256 \times 256$. To learn a dictionary for each image, we randomly selected 30000 blocks of it as the training signals. We repeated each experiment (corresponding to a certain level of noise) 5 times and averaged the results. The final PSNR values are shown in Table 1. The average execution times of K-SVD and PAU-DL were approximately 90 and 30 seconds, respectively.

These results together with those in the previous experiments all suggest the advantage of PAU-DL compared to KSVD.

\section{CONCLUSION}

We proposed a fast and efficient alternative to K-SVD. Our algorithm is based on updating the atoms of the dictionary in parallel using the idea of alternating minimization. As the simulations results on both synthetic and real data showed, our algorithm outperforms K-SVD in both the average execution time and the quality of the results.

\section{REFERENCES}

[1] M. Elad, Sparse and Redundant Representations. Springer, 2010.

[2] D. L. Donoho, "Compressed sensing," IEEE Trans. on Information Theory, vol. 52, no. 4, pp. 1289-1306, April 2006. 
Table 1. Image denoising PSNR results in $\mathrm{dB}$. In each cell two results are reported. Top: results of the K-SVD, and Bottom: results of the PAU-DL. The average execution times of K-SVD and PAU-DL were 90 and 30 seconds, respectively.

\begin{tabular}{||c||c||c||c||c||c||}
\hline \hline$\sigma$, PSNR & House & Boat & Lena & Barb. & Ave. \\
\hline \hline \multirow{2}{*}{$2,42.11$} & 44.48 & 43.66 & 44.50 & 43.96 & 44.15 \\
\cline { 2 - 6 } & 44.50 & 43.67 & 44.53 & 43.98 & 44.15 \\
\hline \hline \multirow{2}{*}{$5,34.16$} & 39.44 & 37.41 & 38.86 & 38.13 & 38.46 \\
\cline { 2 - 6 } & 39.46 & 37.43 & 38.89 & 38.18 & 38.50 \\
\hline \hline \multirow{2}{*}{$10,28.11$} & 36.08 & 33.32 & 35.02 & 34.10 & 34.63 \\
\cline { 2 - 6 } & 36.14 & 33.37 & 35.10 & 34.15 & 34.69 \\
\hline \hline \multirow{2}{*}{$15,24.62$} & 34.44 & 31.04 & 32.85 & 31.83 & 32.54 \\
\cline { 2 - 6 } & 34.51 & 31.08 & 32.91 & 31.86 & 32.59 \\
\hline \hline \multirow{2}{*}{$20,22.11$} & 33.27 & 29.54 & 30.26 & 30.17 & 30.81 \\
\cline { 2 - 6 } & 33.31 & 29.57 & 30.28 & 30.19 & 30.84 \\
\hline \hline \multirow{2}{*}{$25,20.16$} & 32.19 & 28.33 & 30.00 & 29.02 & 29.89 \\
\cline { 2 - 6 } & 32.21 & 28.35 & 30.04 & 29.04 & 29.91 \\
\hline \hline \multirow{2}{*}{$50,14.15$} & 28.07 & 24.81 & 26.14 & 25.33 & 26.09 \\
\cline { 2 - 6 } & 28.08 & 24.85 & 26.16 & 25.34 & 26.11 \\
\hline \hline \multirow{2}{*}{$100,8.12$} & 23.68 & 21.80 & 22.57 & 21.90 & 22.49 \\
\cline { 2 - 5 } & 23.69 & 21.82 & 22.60 & 21.91 & 22.51 \\
\hline \hline
\end{tabular}

[3] M. Elad, M. A. T. Figueiredo, and Y. Ma, "On the role of sparse and redundant representations in image processing," Proceedings of the IEEE, vol. 98, no. 6, pp. 972-982, 2010.

[4] R. Tibshirani, "Regression shrinkage and selection via the lasso," J. Royal. Statist. Soc B., vol. 58, no. 1, pp. 267-288, 1996.

[5] S. Mallat and Z. Zhang, "Matching pursuits with time-frequency dictionaries," IEEE Trans. on Signal Proc., vol. 41, no. 12, pp. 3397-3415, 1993. [Online]. Available: citeseer.ist.psu.edu/mallat93matching.html

[6] D. L. Donoho, M. Elad, and V. Temlyakov, "Stable recovery of sparse overcomplete representations in the presence of noise," IEEE Trans. Info. Theory, vol. 52, no. 1, pp. 6-18, 2006.

[7] H. Mohimani, M. Babaie-Zadeh, and C. Jutten, "A fast approach for overcomplete sparse decomposition based on smoothed $\ell^{0}$ norm," IEEE Trans. on Signal Processing, vol. 57, pp. 289-301, 2009.

[8] S. S. Chen, D. D. Donoho, and M. A. Saunders, "Atomic decomposition by basis pursuit," SIAM Rev., vol. 43, pp. 129-159, 2001.

[9] J. A. Tropp and S. J. Wright, "Computational methods for sparse solution of linear inverse problems," Proceedings of the IEEE, vol. 98, no. 6, pp. 948-958, 2010.

[10] Y. C. Pati, R. Rezaiifar, and P. S. Krishnaprasad, “Orthogonal matching pursuit: recursive function approximation with applications to wavelet decomposition," in In Proc. Asilomar Conf. Signal Syst. Comput., 1993.

[11] D. Needell and J. A. Tropp, "Cosamp: Iterative signal recovery from in-complete and inaccurate samples," Appl. Comput. Harmon. Anal., vol. 26, no. 3, pp. 301321, 2009.

[12] M. Elad, B. Matalon, J. Shtok, and M. Zibulevsky, "A wide-angle view at iterated shrinkage algorithms," in in Proc. SPIE (Wavelet XII), 2007, pp. 26-29.

[13] R. Chartrand and W. Yin, "Iteratively reweighted algorithms for compressive sensing," in IEEE ICASSP, 2008.

[14] M. Elad and M. Aharon, "Image denoising via sparse and redundant representations over learned dictionaries," IEEE Trans. on Image Processing, vol. 15, no. 12, pp. $3736-3745,2006$.

[15] J. Mairal, F. Bach, and J. Ponce, "Task-driven dictionary learning," IEEE Trans. on Pattern Analysis and Machine Intelligence, vol. 34, no. 4, pp. 791-804, 2012.

[16] M. Aharon, M. Elad, and A. Bruckstein, "K-SVD: An algorithm for designing overcomplete dictionaries for sparse representation," IEEE Trans. on Signal Processing, vol. 54, no. 11, pp. 4311-4322, 2006.

[17] R. Rubinstein, M. Zibulevsky, and M. Elad, "Efficient implementation of the K-SVD algorithm using batch orthogonal matching pursuit," Technion University, Tech. Rep., 2008.

[18] K. Skretting and K. Engan, "Recursive least squares dictionary learning algorithm," IEEE Trans. on Signal Processing, vol. 58, pp. $2121-2130,2010$. 